\title{
Tradition und Wandel in der materiellen Kultur der Arrernte und Luritja in Zentralaustralien . Ein Vergleich mit musealen Quellen
}

\section{Birgit Scheps, Kustodin für Australien und Ozeanien am Museum für Völkerkunde zu Leipzig}

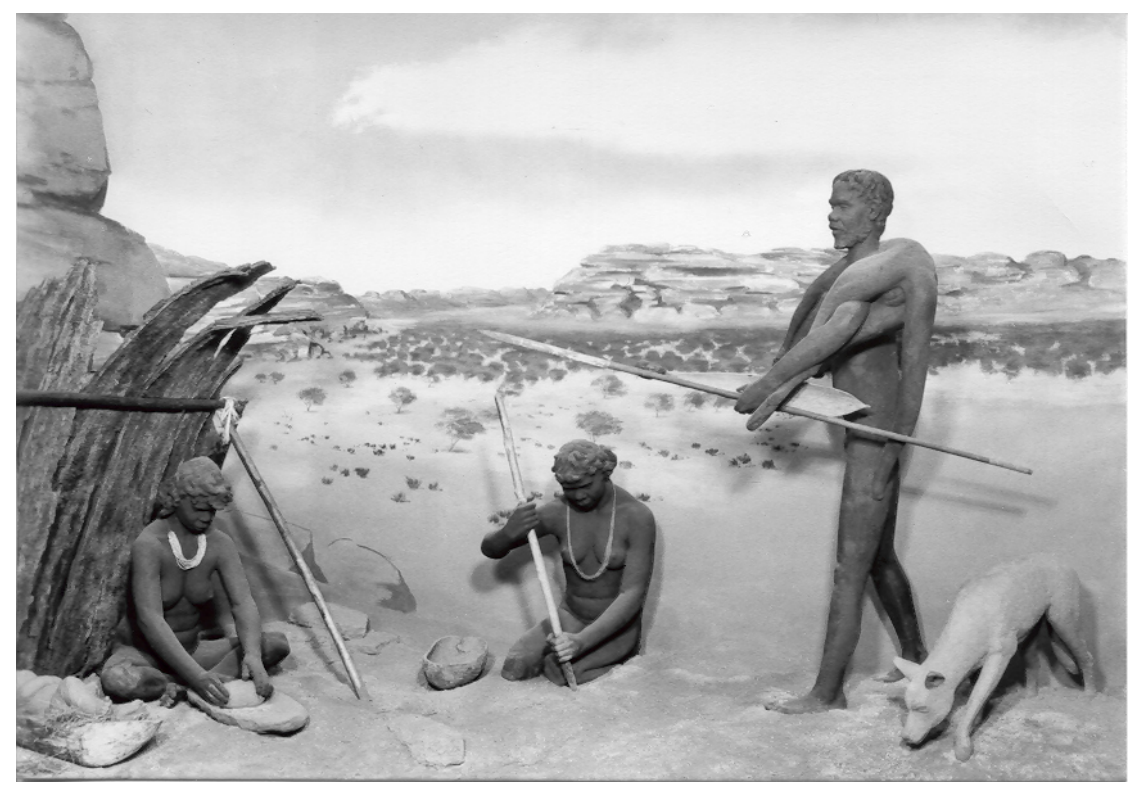

(Postkarte vom Museum für Völkerkunde zu Leipzig über Autorin)

Das Museum für Völkerkunde zu Leipzig bewahrt bedeutende Sammlungen australischer Sachzeugnisse der verschiedenen Ureinwohnervölker. Viele Stücke stammen aus dem 19. Jahrhundert, einer Zeit, in der die Ureinwohner Australiens noch relativ unberührt von der weißaustralischen Kultur lebten. Ebenso finden sich in den Sammlungen auch Zeugnisse, die vom Wandel der Kulturen in Australien in der Zeit der wirtschaftlichen, missionarischen und touristischen Entwicklungen berichten. Gerade in den letzten Jahrzehnten wurden z.B. viele der Communities in Zentralaustralien in Tourismusprojekte eingebunden, sie entwickelten neue Souvenirs und Kunstrichtungen. Zur Sammlungsstrategie unseres Museums für die Region Australien gehört es u.a., auch die neuen Entwicklungen und ihren Einfluß auf die traditionellen Strukturen durch typische Beispiele zu dokumentieren.

Dieses ist nur möglich durch intensive Feldforschungen und Sammeltätigkeit in Australien, die für das Museum für Völkerkunde zu Leipzig in den 1960er und 1970er Jahren durch Prof. F.G.G. Rose in Zentralaustralien und seit den 1990er Jahren von mir durchgeführt worden sind.

Über einen Zeitraum von nunmehr 13 Jahren ermöglichten mir 7 mehrmonatige Aufenthalte bei den Arrernte, Luritja und Warlpiri sowie bei den Tiwi auf Bathurst Island einen intensiven Kontakt, langjährige Freundschaften und dadurch eine enge Zusammenarbeit. Daraus resultieren auch Einblicke in innere Strukturen, Wandlungen, Machtverhältnisse und Entwicklungstendenzen. 
Eine besonders intensive Zusammenarbeit entwickelte sich mit den Arrernte $^{1}$ in und um Mparntwe (Alice Springs) und im Gebiet von Ntaria (Hermannsburg). Die Arrernte unterteilen sich territorial in fünf Gruppen: in die nördlichen, südlichen, östlichen, westlichen und zentralen Arrernte, die wiederum zwei Sprachgruppen angehören: den Eastern und Western Arrernte. Beide Sprachgruppen überlappen sich im Gebiet um Alice Springs und nordwestlich davon. ${ }^{2}$

Sie zählen heute rund 3000 Menschen. Ihr Siedlungsgebiet reicht von Mparntwe (Alice Springs) im Zentrum bis nach Ntaria (Hermannsburg) im Westen und Ltyente Apurte im Osten. Ein großer Teil der traditionellen Siedlungsgebiete wurde in den späten 1970ern ${ }^{3}$ bis in die 1990-er Jahre an die Arrernte zurückgegeben ${ }^{4}$.

Ein Teil von ihnen lebt im Gebiet der Stadt Alice Springs, um die Stadt herum gibt es eine Vielzahl von Camps. Die Mobilität der Arrernte nimmt stetig zu, entsprechend ihrem Bedürfnis, wieder mehr "bush" zu gehen, d.h. viele pendeln in mehr oder weniger großen Abständen zwischen Alice Springs oder auch anderen Ortschaften, zumeist ehemaligen Missionsstationen wie Ntaria (Hermannsburg) oder Tijingala (Santa Teresa) und ihren Bushcamps (outstations), die häufig Tagesmärsche entfernt sind, um auf ihrem Clanland 'traditional' ${ }^{6} \mathrm{zu}$ leben. Vor allem diesen Gruppen gilt mein besonderes Interesse.

Der Schwerpunkt unserer gemeinsamen Arbeit hat eine museale Basis: die Sammlungen aus Zentralaustralien, die von den Missionaren der deutschen lutheranischen Mission im Gebiet von Hermannsburg, ca. $140 \mathrm{~km}$ in nordwestlicher Richtung von Alice Springs entfernt, angelegt wurden.

Zu nennen sind hierbei besonders Carl Strehlow ${ }^{7}$ (dessen Sammlungen vor allem in das damalige Städtische Völker-Museum in Frankfurt am Main ${ }^{8}$ gelangten) und auch Oskar Liebler", dessen intensive "Jagd" nach Sammlungsgegenständen eine Vielzahl von Objekten in die Museen von Leipzig, Stuttgart und Freiburg im Breisgau gelangen ließ. Das Museum

\footnotetext{
${ }^{1}$ Früher übliche Bezeichnung: Aranda, Arunta. Die verschiedenen Schreibweisen dieses Namens gehen auf die Umschrift zurück, mit der entweder deutsche oder englische Muttersprachler den gehörten Namen schriftlich umsetzten. Seit Ende der 1980er Jahre erschienen verbindliche Wörter- und Grammatikbücher, die ein einheitliches Lautsystem für die geschriebenen Arrernte- Sprachen anwenden. Die hier verwendete Schreibweise der ArrernteNamen stützt sich auf die im "Eastern and Central Arrernte to English Dictionary" verwendete.

2 (Latz 1996 : XV)

${ }^{3}$ Der Aboriginal Land Rights (Northern Territory) Act trat 1976 in Kraft. An den Bestrebungen zur Schaffung und Durchsetzung dieses Landrechtsgesetzes hatten die Arrernte einen erheblichen Anteil.

${ }^{4}$ So ist auch das Land, auf dem die Stadt Alice Springs steht, traditionelles Eigentum von Arrernte- Clans. Nach der Rückgabe dieses Landes leaste die Stadtverwaltung das Gebiet von den traditionellen Eigentümern. (Morton: 1999: 329)

5 Der Begriff "Camp" wird als Terminus technicus verwendet, da die Siedlungsweise der einzelnen Clans sehr unterschiedlich ist. Das mit "Camp" umschriebene Spektrum reicht von einer Örtlichkeit mit kleinen Häusern, temporären Camps im trockenen Flussbett des Todd River im Stadtgebiet von Alice Springs bis hin zum viele Kilometer entfernten Lagerplatz im Busch.

${ }^{6}$ Die enge Beziehung der Menschen zu ihrem Land ist dafür Grundlage. Das traditionelle und zeremonielle Wissen, das bei den Arrernte noch sehr lebendig ist, ermöglicht das Leben im Busch. Jahreszeiten, Nahrungsangebot und zeremonielle Zyklen bestimmen den Rhythmus der Wanderungen im Land.

${ }^{7}$ Carl Strehlow kam 1894 in die Mission von Hermannsburg und blieb, mit kleinen Unterbrechungen, bis 1922.

${ }^{8}$ Hier wurden auch durch Moritz Freiherr von Leonhardi die Aufzeichnungen und Interpretationen von Carl Strehlow zur Kultur der Aranda veröffentlicht, die heute Klassiker der Ethnologie Australiens sind. Angeregt durch Leonhardi kamen auch umfangreiche Sammlungen von Strehlow ins Frankfurter Museum. Einige der Stücke gelangten dann später auch nach Leipzig und Berlin.

${ }^{9}$ Oskar Liebler war von 1894 bis 1915 in der Mission von Hermannsburg tätig
} 
für Völkerkunde zu Leipzig erwarb dabei, damals in offensichtlicher Konkurrenz zu Frankfurt, den größten Teil der Sammlungen Lieblers, in Freiburg befinden sich 17 Stücke und das Linden-Museum in Stuttgart besitzt ebenfalls eine solche Sammlung.

Lieblers Objekte stammen aus allen Bereichen des Lebens der Arrernte. Es finden sich Rohstoffe (z.B. Kängurusehnen), Nahrungsmittel (Wurzeln, Insekten), Werkzeuge und Geräte (in verschiedenen Herstellungsstufen), Schmuckgegenstände, Waffen, Alltagsdinge, Spielzeug und auch rituelle Objekte verschiedener Art.

Alle diese Sammlungsstücke wurden stets mit schriftlichen Informationen zur Art des Gebrauches, zu seinen Eigentümern oder Produzenten oder auch $\mathrm{zu}$ den Fund- und Erwerbsorten ins Museum geschickt. Viele dieser Notizen sind erhalten geblieben und heute stellen sie auch für die Arrernte wertvolle Quellen dar.

Die wissenschaftliche Erschließung dieser Quellen wird von mir im Museum vorbereitet. Die vorhandenen Notizen (noch in der alten Kanzleischrift) werden "entziffert" und per Computer erfasst. Dabei ergeben sich bereits thematische Komplexe, die über den materiellen Bezug zum Museumsobjekt hinausgehen: Daten über Wanderbewegungen, Daten personeller Art, Daten über die Zusammensetzung der kleinen wandernden Einheiten, Bezüge zum von ihnen genutzten Territorium, Beziehungen zu benachbarten Gruppen, Einzelheiten zu zeremoniellen Abläufen und vieles mehr.

Auch die objektbezogenen Daten liefern eine Fülle von Informationen über nichtmaterielles Wissen, z.B. über die Zubereitung und Verwendung von im Busch gesammelter Nahrung, über Reife- und Nutzungszeiten von Pflanzen, Kalenderwissen, Kenntnisse über mythische Beziehungen von Personen, Pflanzen, Tieren oder Gegenständen, rituelles und spirituelles Wissen u.v.a. Solche Daten werden dann vor Ort lebendig, und es ist ein Ziel der Arbeit, festzustellen, was von diesem Wissen heute noch - vollständig oder auch nur teilweise - bei den Nachfahren dieser Arrerntegruppen vorhanden ist.

Zum einen hilft dabei die eigene Beobachtung (z.B.: Welche Gegenstände werden heute im Buschcamp benutzt? Haben sich die Materialien und/ oder die Herstellungsweise verändert? Welche Dinge sind verschwunden und wodurch wurden sie ersetzt? Kennt man ihre Vorgänger trotzdem noch und was weiß man darüber? Wer lebt im Camp zusammen? Wer wandert mit wem? Wie ändert sich die Zusamensetzung der Familien?), aber am wichtigsten sind die gemeinsamen Diskussionen der in den alten Quellen getroffenen Aussagen. Als Frau sind mir vor allem die Frauen Gesprächs- und Aktionspartner, z.B. lese ich ihnen die Notizen vor, die Liebler zu einem Sammlungsstück gemacht hat. Sehr oft beginnen diese Notizen mit der Nennung des "Arandawortes" für diesen Gegenstand. Schon hier zeigt sich dann, daß er auch Benennungen aus dem Luritja oder auch dem Anmatyerre aufführt, die man ihm damals sagte - je nachdem, wen er gefragt hat und was die Muttersprache dieses Informanten war. Auch heute leben in den gemeinsam wandernden Gruppen Menschen verschiedener Muttersprachen zusammen, man ist - damals wie heute - mehrsprachig, man ist miteinander blutsverwandt oder totemistisch verbunden, und materielle und spirituelle Kooperation ist genauso von lebenswichtiger Bedeutung wie früher.

Beschreibung und Angaben über den Gebrauch der Gegenstände sind bei Liebler oft mit persönlichen Kommentaren oder Wertungen ergänzt. Diese Anmerkungen lösen bei den Frauen oft sehr intensive Diskussionen aus. Um den Wahrheitsgehalt einer Aussage zu klären, versuchen sie z.B., sich an lange zurückliegende Vorgänge zu erinnern, die mit den 
geschilderten Sachverhalten korrespondieren, oder von denen sie u.U. von ihren Eltern oder Großeltern gehört haben. So kommen viele "stories" wieder ins Bewußtsein, die dann auch an die Kinder, die sehr oft als Zuhörer und Lernende bei den Gesprächen dabei sitzen, weitergegeben werden. Geschichte wurde und wird oral tradiert und den "stories" kommt dabei eine sehr wichtige Bedeutung im kollektiven Gedächtnis zu.

Viele Aussagen lassen sich relativ einfach überprüfen: Dinge, die noch im Alltag verwendet werden, kann ich mir vorführen und erläutern lassen. Ich kann beobachten, wie man sie herstellt, wie und wo man das Material dafür findet und wie die Arbeitsgänge genau ablaufen. Solche Gegenstände werden dann auch für das Museum gesammelt, und sie ergeben nun eine "Parallele" zur historischen Sammlung. Hier wird dann im Vergleich der historischen Objekte mit den Neuerwerbungen sehr deutlich, wie diese alte Kultur sehr flexibel auf fremde Einflüsse, Umwälzungen und Veränderungen reagiert hat und wie sie traditionellen Werte bewahren konnte.

Diese Ausführungen beleuchten nur einen kleinen Teil des Spektrums der Themen und des Handlungsrahmens meines Projektes. Sie sollen exemplarisch zeigen, welche immense Bedeutung den materiellen Zeugnissen in den ethnologischen Sammlungen als lebendige historische Quelle zukommt, wenn man sie zum "Sprechen" bringt.

\section{Literatur}

Brooks, David: 1996. The Arrernte Landscape. A Guide to the Dreaming Tracks and sites of Alice Springs. Alice Springs: IAD Press

Henderson, J. / Dobson, V.: 1994. Eastern and Central Arrernte to English Dictionary. Alice Springs: IAD Press

Latz, Peter: 1996. Bushfires \& Bushtucker. Aboriginal Plant Use in Central Australia. Alice Springs: IAD Press

Morton, John: 1999. Arrernte. In: Richard B. Lee und Richard Daly (Hg.) The Cambridge Encyclopedia of Hunters and Gatherers. 329 - 334. Cambridge: Cambridge University Press

Scheps, Birgit: 2000. Der Kontinent der Träume. Leipzig: Museum für Völkerkunde zu Leipzig

Scheps, Birgit: 2002. Der Traum von der Raupe: Eßbare Raupen bei den Arrernte in Zentralaustralien. In: Wirtschaften. Veröffentlichungen des Instituts für Ethnologie der Universität Leipzig, Reihe: Ethnographie, Bd.2, Escher Verlag Gehren

Spencer, B. / Gillen, F. J.: 1968. The Native Tribes of Central Australia. New York: Dover (Reprint der Ausgabe von 1899)

Strehlow, Carl: 1908. Mythen, Sagen und Märchen des Loritja-Stammes. Die totemistischen Vorstellungen und die Tjurunga der Aranda und Loritja. In: Die Aranda- und Loritja-Stämme in Zentral-Australien. Veröffentlichungen aus dem Städtischen Völker-Museum Frankfurt am Main, Bd.I, II.Teil

Strehlow, Carl: 1910. Die totemistischen Kulte der Aranda- und Loritja-Stämme. In: Die Arandaund Loritja-Stämme in Zentral-Australien. Veröffentlichungen aus dem Städtischen VölkerMuseum Frankfurt am Main, Bd.III, I.Abteilung

Strehlow, Theodor G.H.: 1947. Aranda traditions. Melbourne: Melbourne University Press

Strehlow, Theodor G.H.: 1971. Songs of Central Australia. Sydney: Angus \& Robertson 1978 Journey to Horseshoe Bend. Adelaide: Rigby

Turner-Neal, Margaret-Mary/ Henderson, John: 1994. Bush Foods. Arrernte Foods from Central Australia. Alice Springs: IAD Press 